\title{
CT for Pediatric, Acute, Minor Head Trauma: Clinician Conformity to Published Guidelines
}

\author{
L.L. Linscott, M.M. Kessler, D.R. Kitchin, K.S. Quayle, C.F. Hildebolt, R.C. McKinstry, and S. Don
}

\begin{abstract}
BACKGROUND AND PURPOSE: In 2001, pediatric radiologists participating in a panel discussion on CT dose reduction suggested that approximately $30 \%$ of head CT examinations were performed unnecessarily. With increasing concern regarding radiation exposure to children and imaging costs, this claim warrants objective study. The purpose of this study was to test the null hypothesis that $30 \%$ of head CT studies for clinical evaluation of children with acute, minor head trauma do not follow established clinical guidelines.
\end{abstract}

MATERIALS AND METHODS: Retrospective review of 182 consecutive patients with acute, minor head trauma from February 2009 to January 2010 at a tertiary care children's hospital emergency department was performed, and clinician adherence to published clinical guidelines for children younger than 2 years and children 2-20 years of age was determined. The binomial test was used for a null hypothesis of $30 \%$ unnecessary examinations against the actual percentage of head CTs deemed unnecessary on the basis of established guidelines. Statistical testing was performed for children younger than 2 years and 2-20 years of age.

RESULTS: For children younger than 2 years of age, 2 of 78 (2.6\%; $95 \% \mathrm{Cl}, 0.5 \%-8.3 \%)$ and, for children $2-20$ years of age, 12 of 104 (11.5\%; $\mathrm{Cl}$, $6.4 \%-18.7 \%)$ did not conform to established guidelines. These percentages were significantly less than the hypothesized value of $30 \%$ $(P<.001)$.

CONCLUSIONS: Clinician conformity to published guidelines for use of head CT in acute, minor head trauma is better than suggested by a 2001 informal poll of pediatric radiologists.

ABBREVIATIONS: ALARA $=$ As Low As Reasonably Achievable; $\mathrm{Cl}=$ confidence interval

$\mathbf{T}$

raumatic brain injury is an important clinical problem for children in the United States. Between 2002 and 2006 for children 0-14 years of age, traumatic brain injuries resulted in 473,947 emergency department visits, 35,136 hospitalizations, and 2174 deaths. Of these, approximately 50\% were related to falls, and $90 \%$ were discharged to home. ${ }^{1} \mathrm{CT}$ of the head plays an increasingly important role in the triage of these patients, as evidenced by the steady nationwide increase in CT use for head

Received March 19, 2012; accepted after revision August 23.

From the Mallinckrodt Institute of Radiology (L.L.L., M.M.K., D.R.K., C.F.H., R.C.M. S.D.), Washington University School of Medicine, St. Louis, Missouri; and Departments of Radiology (R.C.M., S.D.) and Pediatrics (K.S.Q.), St. Louis Children's Hospital, St. Louis, Missouri.

Paper previously presented at: American Society of Neuroradiology Annual Meeting and the Foundation of the ASNR Symposium, April 21-26, 2012; New York New York.

Please address correspondence to Luke L. Linscott, MD, Mallinckrodt Institute of Radiology, 510 S Kingshighway Blvd, St. Louis, MO 63110; e-mail:

luke.linscott@gmail.com

http://dx.doi.org/10.3174/ajnr.A3366 trauma during the past decade. The percentage of emergency department visits for head trauma resulting in CT examinations rose steadily from $12 \%$ in 1997 to $34 \%$ in $2008 .^{2}$ Increased awareness of the potential long-term hazards of radiation, particularly in children, ${ }^{3-5}$ has engaged physicians and regulators in attempts to decrease CT use without compromising patient care. ${ }^{6,7}$

In August 2001, a multidisciplinary group, including 40 pediatric radiologists, gathered for the ALARA conference. While discussing means of lowering the overall CT dose to the pediatric population, the pediatric radiologists were informally asked what percentage of head and body CT examinations were performed unnecessarily. The group suggested that approximately $30 \%$ of head CT examinations were performed unnecessarily. ${ }^{8} \mathrm{With}$ increasing concern regarding radiation exposures to children and imaging costs, it is important to determine the extent to which unnecessary CT examinations are being performed. In this study, we retrospectively reviewed CT cases to determine clinician conformity to guidelines for CT examinations for the clinical evaluation of infants and children with acute, minor, closed-head trauma. ${ }^{9,10}$ The null hypothesis was that $30 \%$ of head CT studies 
for clinical evaluations of infants and children with acute, minor, closed-head trauma do not follow established guidelines. ${ }^{8}$

\section{MATERIALS AND METHODS}

From 2001 through 2010 for a large tertiary-care children's hospital, data were collected and plots were made for the number of emergency department patients and the number of head CT examinations. For February 2009 to January 2010, retrospective reviews of 1000 consecutive head CT examinations from the emergency department were performed. Our institutional review board reviewed and approved the study.

The clinical presentation of each patient was reviewed to determine the indication for the examination. Of the 1000 head CTs reviewed, 523 were performed for trauma. The emergency department and inpatient records of these 523 patients $(n=127$ for $0-24$ months of age; $n=396$ for $2-20$ years of age) with head trauma were reviewed in detail to determine whether the cases met published inclusion criteria (guidelines) for acute, minor, closed-head trauma. ${ }^{9,10}$ Of these, 78/127 (61\%) patients younger than 2 years and 104/396 (26\%) patients 2-20 years met the criteria for acute minor head trauma.

We used 2 age-specific clinical guidelines: 0- to 24-month guidelines published in Pediatrics in 2001, ${ }^{9}$ and 2- to 20-year guidelines published in Pediatrics in $1999 .{ }^{10}$ Inclusion criteria for the study were the following: 1) head CT scan performed, and 2) patient presenting with acute minor head trauma. "Acute minor head trauma" was defined as an isolated minor closed-head injury occurring in an otherwise neurologically healthy child. Exclusion criteria varied significantly between the 2 published guidelines. The specific exclusion criteria and the number of patients in each category are found below.

Exclusion criteria for the 0 - to 24-month algorithm included the following: concern for abuse or neglect during initial evaluation $(n=31)$, multiple trauma $(n=7)$, prior outside hospital head CT-prompted evaluation $(n=6)$, pre-existing neurologic disorder $(n=2)$, penetrating injury $(n=2)$, birth trauma $(n=1)$, bleeding diathesis $(n=0)$, and previous intracranial surgery $(n=$ 0). ${ }^{9}$ Exclusion criteria for the 2- to 20 -year algorithm included the following: known or suspected cervical spine injury $(n=93)$, multiple trauma $(n=92)$, initial evaluation $>24$ hours after injury $(n=38)$, suspected intentional head trauma (eg, assault) $(n=34)$, abnormal mental status at the initial examination or abnormal or focal findings on neurologic examination $(n=32)$, pre-existing neurologic disorders potentially aggravated by trauma (such as arteriovenous malformations or shunts) ( $n=$ 27 ), loss of consciousness of $>1$ minute or unobserved loss of consciousness $(n=22)$, physical evidence of skull fracture $(n=$ 18 ), prior outside hospital head CT-prompted evaluation $(n=$ $11)$, presence of drugs or alcohol $(n=4)$, history of bleeding diatheses $(n=2)$, or a language barrier $(n=1) .{ }^{10}$ Of the 292 patients $2-20$ years of age who were excluded, 82 had $>1$ clinical parameter that excluded them from guideline application. If a specific clinical parameter for exclusion from clinical guideline application was not mentioned in the medical record, it was assumed that this parameter was not present. For instance, if concern for abuse or neglect was not explicitly stated in the medical

record, it was assumed that no concern existed. No patient was excluded due to deficiencies in the medical record.

The emergency department and inpatient records were evaluated in detail by 2 diagnostic radiology residents in their fourth year of postgraduate training. There was no overlap in record evaluation. The results of CT examinations came exclusively from written reports. Simple skull fracture was defined as a nondepressed fracture with no associated intracranial injury. Intracranial injury was defined as intracranial hemorrhage, contusion, or cerebral edema.

After identifying those patients whose presentation qualified as acute, minor head trauma ( $n=127$ for $0-24$ months of age; $n=396$ for 2-20 years of age), these patient records were reviewed to assess whether the emergency department physicians conformed to the published guidelines for appropriate use of CT to evaluate possible intracranial injury. Instances of clinician nonconformity to guidelines were identified, and specific points of departure were recorded. An unnecessary CT was defined as a CT examination performed when imaging was not recommended according to the clinical guideline.

The null hypothesis was that $30 \%$ of head CT studies for clinical evaluation of infants and children with acute, minor, closedhead trauma do not follow established guidelines. ${ }^{8}$ Exact $P$ values for the binomial test were calculated to test this null hypothesis (ie, to determine whether the observed proportion in the population was different from the theoretic value [constant] of 30\%). Tests were performed for children younger than 2 years of age and for children 2-20 years of age. The statistical power of the tests was calculated. Blyth-Still-Casella 95\% confidence intervals were determined for proportions. Statistical analyses were performed with StatXact-9 Statistical Software for Exact Nonparametric Inference (Cytel, Cambridge, Massachusetts).

\section{RESULTS}

In 2001, nine hundred sixty-one head CT examinations were performed in our emergency department. The number of annual examinations increased each year until 2007, with 1453 examinations performed that year. After 2007, the annual number of examinations steadily dropped, with only 956 examinations performed in 2010 (Fig 1). The number of emergency department visits remained steady during this period. No specific emergency or radiology department initiatives to reduce CT use were in place during the time of this study.

Of the 1000 head CT examinations reviewed in our study, we found that most examinations, 523 (52\%; Blyth-Still-Casella 95\% confidence intervals, 49\%-55\%), were performed for trauma. In the 0 - to 24 -month age group, $127 / 218$ (58\%; 95\% CI, $52 \%-65 \%)$ examinations were performed for trauma, and in the 2 - to 20 -year age group, $396 / 770$ (51\%; 95\% CI, 48\%-55\%) were performed for trauma (Fig 2).

When patient records from all trauma-related CT examinations were reviewed to determine whether the patient qualified for application of the clinical guidelines, we obtained the following results: $78 / 127(61 \%$; 95\% CI, 52\%-70\%) patients 0-24 months of age qualified for use of the guideline, and 104/396 (26\%; $95 \%$ CI, $22 \%-31 \%$ ) patients $2-20$ years of age qualified for use of the guideline. 


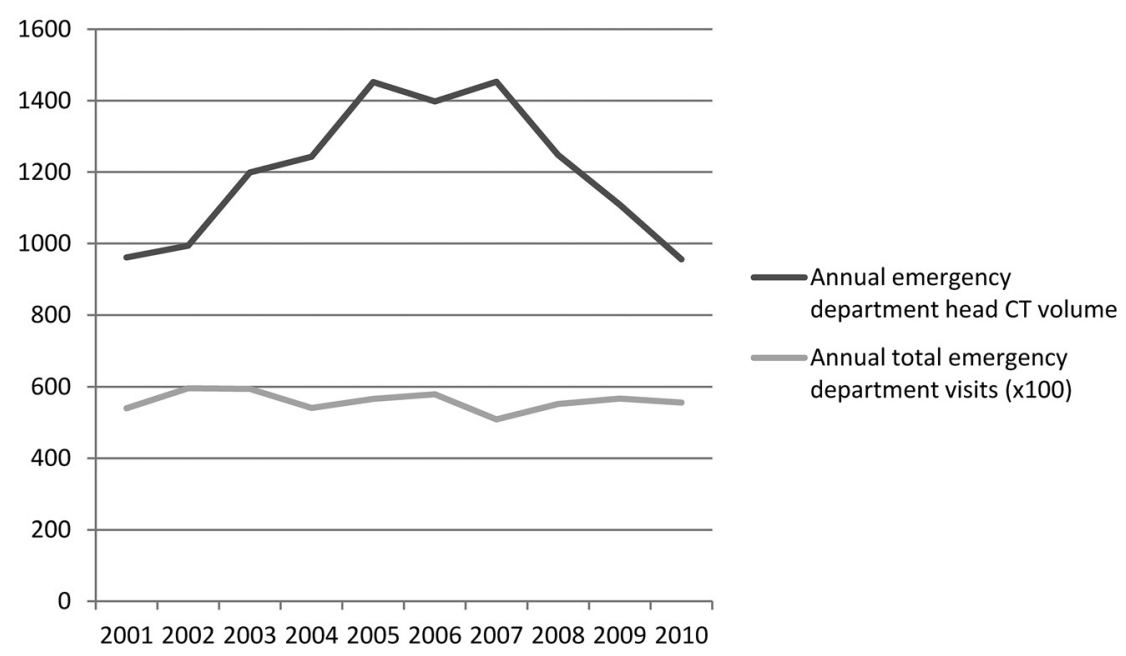

FIG 1. Head CT use (examinations/year) and annual total emergency department visits $(\times 100)$ at our institution from 2001 to 2010.

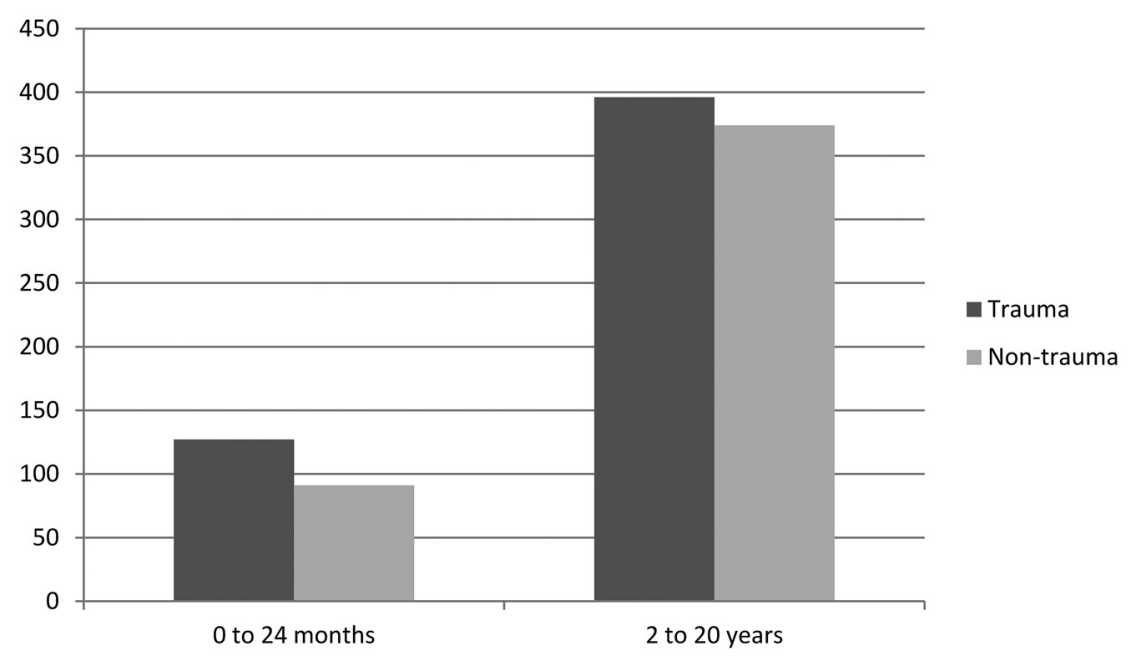

FIG 2. Breakdown of 1000 head CT examinations performed because of trauma or other causes.

For ages $0-24$ months, nonconformity to clinical guidelines occurred in 2 of 78 (2.6\%; 95\% CI, $0.5 \%-8.3 \%)$ patients. For patients 2-20 years of age, nonconformity to clinical guidelines occurred in 12 of 104 patients (11.5\%; 95\% CI, 6.4\%-18.7\%) (Fig 3). These percentages were significantly less than the hypothesized value of $30 \%(P<.001)$. The statistical power of these tests was $>99 \%$.

For ages 0-24 months, results of head CT examinations reported in the medical record are listed in Table 1, with 11 patients presenting with acute minor head trauma having isolated simple skull fractures and 11 patients having intracranial injuries. For ages 2-20 years, CT results are listed in Table 2, with 1 patient presenting with acute minor head trauma having an isolated simple skull fracture and 2 patients having intracranial injuries. An example of a skull fracture with intracranial hemorrhage in a 5-month-old girl is presented in Fig 4.

\section{DISCUSSION}

Many physicians, regulators, and patients are concerned that CT is being overused, particularly for pediatric patients for whom risks from ionizing radiation are greater than those in adults. ${ }^{11}$
Concerns are supported by studies demonstrating increased CT use with time $\mathrm{e}^{12-14}$ and/or studies that indicate that the overall diagnostic yield from $\mathrm{CT}$ examinations (for specific indications) is decreasing with time. ${ }^{15}$ Overuse is one of the concerns that have spurred the creation of social media campaigns (such as the Image Gently campaign of the Alliance for Radiation Safety in Pediatric Imaging) to reduce unnecessary imaging procedures that use ionizing radiation.

The head CT rate performed by our emergency department does not support a trend for increasing CT use. The rate has decreased from 1453 in 2007 to 956 in 2010-a 34\% reduction even though the emergency department visit rate remained stable during this period. No specific emergency or radiology department initiatives to reduce $\mathrm{CT}$ use were in place during the time of this study. While we did not evaluate the percentage of conformity to guidelines for head CT in 2007, we speculate that increased awareness of the risk of CT from campaigns such as Image Gently has reduced the number of unnecessary CT examinations ordered.

In the August 2001 ALARA conference, during a panel discussion session entitled "Helical CT and Cancer Risk," an informal poll of approximately 40 pediatric radiologists suggested that as many as $30 \%$ of body and head CT examinations were performed unnecessarily. ${ }^{8}$ There are, however, few studies that document clinician conformity to established CT standards of care (such as published guidelines and/or diagnostic algorithms). We, therefore, retrospectively determined the extent to which established guidelines were followed by clinicians who used CT for pediatric acute, minor head trauma and found that $>88 \%$ of CT examinations conform to CT standards of care. Our findings do not support government agencies, health insurance companies, and radiologists who suggest that head CT is overused for the evaluation of acute, minor head trauma in children. At our institution in the age of Image Gently, conformity to guidelines is high.

On the basis of our study, we think that current guidelines/ algorithms for use with CT imaging have limitations. In our study, $75 \%$ of 2 - to 20 -year-old patients with head trauma had conditions (such as unwitnessed injuries or pre-existing neurologic conditions), that excluded them from clinical guideline application. This was not the case for 0 - to 24-month-old patients with head trauma, in whom only $39 \%$ had presenting symptoms or conditions that excluded them from guideline application. We believe that the disparity between these 2 groups is in the strictness of the exclusion criteria for each guideline; the 2- to 20-year 


\section{0 to 24 months}
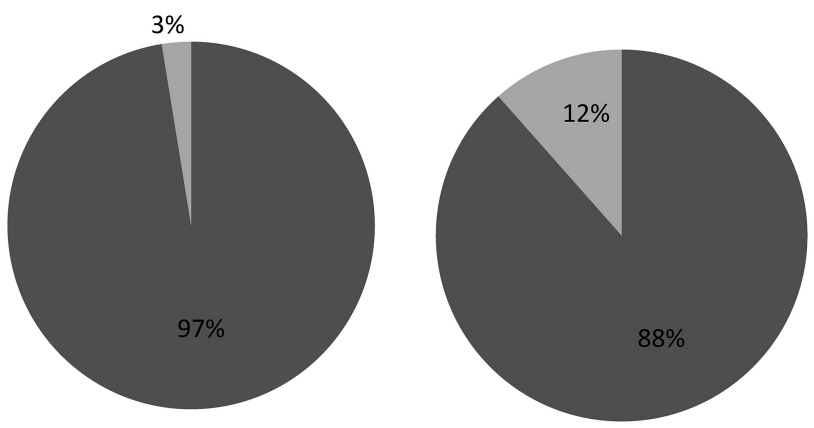

- Conformed to guidelines

FIG 3. Percentage of cases in which emergency department clinicians conformed to the diagnostic guidelines.

Table 1: Zero- to 24-month-old patients with minor head trauma who qualified for use of the clinical guidelines: CT imaging findings for acute, minor head trauma

\section{CT Imaging Findings} No. of Patients

Conformed to the algorithm with 54 normal head CT findings

Conformed to the algorithm with simple skull fractures

Conformed to the algorithm with intracranial injury

Did not conform to the algorithm with normal head CT findings (ie, "unnecessary CT")

Did not conform to the algorithm with abnormal head CT findings

Table 2: Two- to 20-year-old patients with minor head trauma who qualified for use of the clinical guidelines: CT imaging findings for acute, minor head trauma

CT Imaging Findings

No. of Patients

Followed the algorithm with normal 89 head CT findings

Followed the algorithm with simple skull fractures

Followed the algorithm with intracranial injury

Did not follow the algorithm with normal head CT findings

(ie, "unnecessary CT")

Did not follow the algorithm with abnormal head CT findings

guideline was much stricter in its exclusion criteria. Recently, a prospective cohort study of children with head injury was performed to identify patients at "very low risk of clinically important brain injury," and guidelines for imaging were brought upto-date. ${ }^{16}$ We consider these guidelines less restrictive in the use of CT for the evaluation of acute, minor head trauma than the guidelines used in our study. Many published guidelines do not account for unique patient/environmental factors that must be dealt with by the medical team on a case-by-case basis. Effort should be made to create clinical guidelines that apply to most patients.

There were no poor outcomes in our study. We found that a substantially higher proportion of examinations in the 0 - to 24-

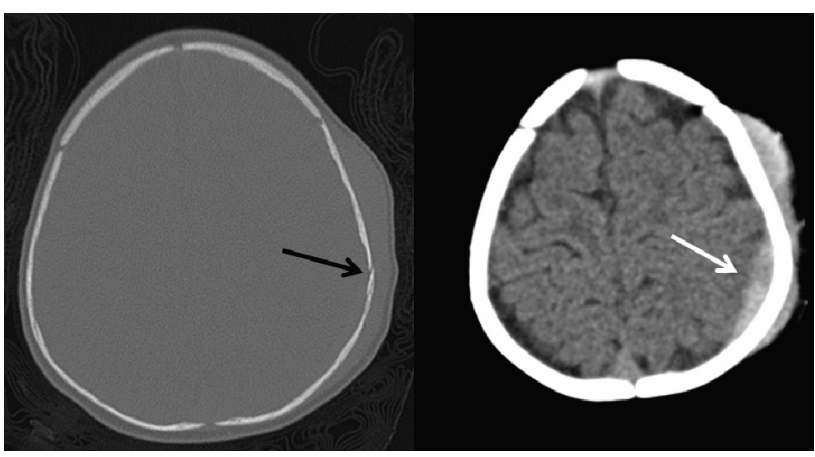

FIG 4. CT of the head in a 5-month-old girl who fell 3-4 feet off a bed onto a wooden floor. Parents reported no loss of consciousness or change in behavior. Physical examination revealed a left parietal scalp hematoma. Head CT showed a nondisplaced left parietal bone fracture (black arrow) with small underlying extra-axial fluid collection, likely an epidural hematoma (white arrow).

month age group yielded findings of intracranial injury: 11/78 (14\%) versus $2 / 104(2 \%)$ in the 2 - to 20 -year age group. This may be due to the insensitivity of clinical factors in identifying intracranial injury in the 0 - to 24 -month age group and/or an increased predisposition of this age group to intracranial injury in the setting of minor head trauma. ${ }^{17,18}$ This finding underscores the importance of CT in triaging this young group of patients.

Our study has several weaknesses. Our data were collected from a tertiary care children's hospital and may not be generalizable to emergency departments in other countries, community hospitals, or areas with less access to CT. For instance, in Scandinavia, it was found that that $27 \%$ of head CT examinations were unnecessary on the basis of Scandinavian guidelines. ${ }^{19}$ Another weakness of our study is that it is focused on a single emergency department condition - acute, minor head trauma in infants and children. There may be various degrees of conformity to imaging guidelines for various conditions. For instance, it was recently found that for cervical spine trauma, $\geq 20 \%$ of CT examinations could be avoided if clinical guidelines were followed. ${ }^{20}$

\section{CONCLUSIONS}

On the basis of this retrospective study, clinician conformity to published guidelines for use of head CT in acute, minor head trauma is better than suggested by a 2001 informal poll of pediatric radiologists for a tertiary care pediatric children's hospital. While clinical guidelines may be an important tool in ensuring appropriate use of medical imaging, unique patient characteristics often exclude a large proportion of patients from enrollment in diagnostic algorithms. Making guidelines more inclusive should be encouraged.

Disclosures: Robert C. McKinstry-UNRELATED: Consultancy: Siemens Healthcare Comments: I was paid as an actor in a TV commercial "Somewhere in America" featuring the Siemens PET/MRI scanner, Travel/Accommodations/Meeting Expenses Unrelated to Activities Listed: Siemens Healthcare, Comments: presented work done with the PET/MRI scanner at May 2012 symposium of the International Society for Magnetic Resonance in Medicine. Steven Don-UNRELATED: Consultancy: Siemens, Payment for Lectures (including service on Speakers Bureaus): Siemens honorarium (approximately $\$ 2000$, less some expenses) for a talk at a Siemens meeting in July 2011 regarding digital radiography unrelated to this CT publication, Patents (planned, pending, or issued): Carestream Health, Comments: image-noise simulator software patent unrelated to this $\mathrm{CT}$ publication. Carestream Health purchased the patent.

AJNR Am J Neuroradiol 34:1252-56 Jun 2013 www.ajnr.org 


\section{REFERENCES}

1. Faul M, Xu L., Wald MM, et al. Traumatic Brain Injury in the United States: Emergency Department Visits, Hospitalizations and Deaths, 2002-2006. Atlanta, Georgia: Centers for Disease Control and Prevention; 2010

2. Larson DB, Johnson LW, Schnell BM, et al. Rising use of CT in child visits to the emergency department in the United States, 1995-2008. Radiology 2011;259:793-801

3. Frush DP, Donnelly LF, Rosen NS. Computed tomography and radiation risks: what pediatric health care providers should know. Pediatrics 2003;112:951-57

4. Brenner DJ, Hall EJ. Computed tomography: an increasing source of radiation exposure. $N$ Engl J Med 2007;357:2277-84

5. Smith-Bindman R. Is computed tomography safe? $N$ Engl J Med 2010;363:1-4

6. International Atomic Energy Agency. Dose Reduction in CT while Maintaining Diagnostic Confidence: A Feasibility/Demonstration Study. IAEA-TECDOC-1621. Vienna, Austria: International Atomic Energy Agency; 2009

7. Brenner DJ, Hricak H. Radiation exposure from medical imaging: time to regulate? JAMA 2010;304:208-09

8. Slovis TL, Berdon WE. Panel discussion. Pediatr Radiol 2002;32: $242-44$

9. Schutzman SA, Barnes P, Duhaime AC, et al. Evaluation and management of children younger than two years old with apparently minor head trauma: proposed guidelines. Pediatrics 2001;107: 983-93

10. The management of minor closed head injury in children: Committee on Quality Improvement, American Academy of Pediatrics. Commission on Clinical Policies and Research, American Academy of Family Physicians. Pediatrics 1999:104:1407-15
11. Committee on the Biological Effects of Ionizing Radiation. Health Effects of Exposure to Low Levels of Ionizing Radiation. BEIR V. Washington, DC: National Academy Press; 1990

12. Gonzales JM. Imaging professionals' beliefs on overutilization of CT and MRI exams. Radiol Manage 2011;33:41-46

13. Armao D, Semelka RC, Elias J Jr. Radiology's ethical responsibility for healthcare reform: tempering the overutilization of medical imaging and trimming down a heavyweight. J Magn Reson Imaging 2012;35:512-17

14. Hendee WR, Becker GJ, Borgstede JP, et al. Addressing overutilization in medical imaging. Radiology 2010;257:240-45

15. Weir ID, Drescher F, Cousin D, et al. Trends in use and yield of chest computed tomography with angiography for diagnosis of pulmonary embolism in a Connecticut hospital emergency department. Conn Med 2010;74:5-9

16. Kuppermann N, Holmes JF, Dayan PS, et al. Identification of children at very low risk of clinically-important brain injuries after head trauma: a prospective cohort study. Lancet 2009;374:1160-70

17. Gruskin KD, Schutzman SA. Head trauma in children younger than 2 years: are there predictors for complications? Arch Pediatr Adolesc Med 1999;153:15-20

18. Greenes DS, Schutzman SA. Occult intracranial injury in infants. Ann Emerg Med 1998;32:680-86

19. Heskestad B, Baardsen R, Helseth E, et al. Guideline compliance in management of minimal, mild, and moderate head injury: high frequency of noncompliance among individual physicians despite strong guideline support from clinical leaders. J Trauma 2008:65: 1309-13

20. Griffith B, Bolton C, Goyal N, et al. Screening cervical spine CT in a level I trauma center: overutilization? AJR Am J Roentgenol 2011; 197:463-67 